\title{
Introduction to "Quantitative bounds of convergence for geometrically ergodic Markov Chain in the Wasserstein distance with application to the Metropolis adjusted Langevin algorithm" by A. Durmus, É. Moulines
}

\author{
Heikki Haario
}

Published online: 6 December 2014

(C) Springer Science+Business Media New York 2014

The Wasserstein distance between probability distributions might be intuitively described as a minimal effort required to map one distribution onto another. The concept has a long history with connections to optimal transport theory. However, the applications on convergence properties of Markov chains are more recent. The present paper contains interesting theoretical work applying recently developed techniques based on the Wasserstein metric for analysing the rate of convergence of geometrically ergodic Markov chains. Bounds on the Wasserstein distances are also based on a drift condition but minorization conditions are replaced by the existence of a coupling set, together with appropriate conditions on the transition kernel. A 'natural' coupling for MCMC algorithms is achieved simply by running two versions of an algorithm with the same random numbers. The main results of the paper can be useful in general when quantifying the (worst-case) convergence of MCMC algorithms to the equilibrium. It appears that at least in certain cases the Wasserstein techniques can provide much tighter upper bounds for the rate of convergence than earlier results which are based on drift and minorization inequalities.
The authors apply their results to the exponential integrator-Metropolis adjusted Langevin algorithm (EI-MALA). They consider an ill-conditioned Bayesian linear inverse problem and demonstrate how their approach scales nicely with dimension, consistent with the theory. In addition, they show that in this example EI-MALA is significantly better than the alternatives tested, pre-conditioned Crank Nicolson and basic MALA. 